Z Rheumatol 2016 $75: 22-31$

DOI 10.1007/s00393-015-0034-6

Online publiziert: 14. Januar 2016

(c) Springer-Verlag Berlin Heidelberg 2015

\section{Redaktion}

H.-P. Tony, Würzburg

CrossMark

\author{
M. Schmalzing ${ }^{1} \cdot$ A. Strangfeld ${ }^{2} \cdot$ H.-P. Tony ${ }^{1}$ \\ 1 Rheumatologie/klinische Immunologie, Medizinische Klinik und Poliklinik 2, Zentrum Innere Medizin, \\ Universitätsklinikum Würzburg, Würzburg, Deutschland \\ ${ }^{2}$ Programmbereich Epidemiologie, Gruppe Pharmakoepidemiologie, DRFZ - Deutsches Rheuma- \\ Forschungszentrum, Berlin, Deutschland
}

\title{
Medikamentöse Therapie der rheumatoiden Arthritis bei Malignomanamnese
}

\section{Epidemiologische Daten}

Die langjährige Betreuung und medikamentöse Behandlung sind für Patienten mit rheumatologischen Erkrankungen und insbesondere mit rheumatoider Arthritis (RA) charakteristisch. Gleichzeitig nimmt durch den demografischen Wandel und die zunehmenden therapeutischen Optionen der Anteil von älteren Patienten, die eine kontinuierliche DMARD („disease modifying antirheumatic drug")-Therapie erhalten oder benötigen, stetig zu. Damit sieht sich der Rheumatologe immer häufiger mit der Komorbidität einer malignen Erkrankung konfrontiert, die so gut wie immer schwierige Entscheidungen in Bezug auf die weitere Therapieplanung mit sich bringt.

Das Auftreten eines Malignoms verschiebt meistens die Priorität des Patienten weg von der rheumatisch entzündlichen Erkrankung und deren Therapie hin zum Malignom, da dieses viel eher als unmittelbare Bedrohung für das Leben wahrgenommen wird. Nach erfolgreicher Therapie eines Malignoms oder in der Phase der Remission rücken rheumatische Beschwerden und die durch sie verursachten Funktionsbeeinträchtigungen jedoch wieder in den Mittelpunkt. Da aber auch die insuffiziente Einstellung einer rheumatisch entzündlichen Erkrankung nicht nur zu Invalidisierung führt, sondern auch mit einer erhöhten Mortalität einhergeht, ist die Fragestellung hochrelevant, inwieweit eine DMARDTherapie bei aktuellem oder stattgehab- tem Malignom mit akzeptabler Sicherheit möglich ist.

Bei dieser Übersichtsarbeit soll der Fokus auf soliden Malignomen und nicht auf hämatoonkologischen Systemerkrankungen liegen.

\section{Methodik}

Zur Identifikation der verwendeten Artikel wurde eine PubMed-Recherche mit folgenden Suchbegriffkonstellationen durchgeführt: „(immunosuppression OR (rheumatoid arthritis) OR methotrexate OR leflunomide OR sulfasalazine OR hydroxychloroquine OR Ciclosporin OR Etanercept OR Infliximab OR Adalimumab OR Golimumab OR Certolizumab OR Anakinra OR Abatacept OR Tocilizumab OR Rituximab) AND (cancer OR malignancy)“. Ausgewählt wurden Originalarbeiten, Therapieempfehlungen und Übersichtsartikel, die Aussagen zum Risiko von De-novo-Malignomen oder von Malignomrezidiven bei Patienten mit RA unter DMARD-Therapie erlauben. Artikel, die in Übersichtsarbeiten zusammengefasst sind, wurden nicht als Einzelartikel aufgenommen.

Außerdem wurden die Abstract-Archive des ACR (American College of Rheumatology)- und des EULAR (European League against Rheumatism)-Kongresses von 2013 bis 2015 mit den Suchbegriffen „cancer“ und „malignancy“ nach dem gleichen Kriterium durchsucht.

\section{Problematische Datenlage}

Bei Empfehlungen zur DMARD-Therapie bei Tumoranamnese kann man sich bislang nicht auf eine suffiziente Datenlage stützen. In den Fachinformationen der betreffenden Medikamente wird entweder nur sehr allgemein darauf verwiesen, dass z. B. bei RA das Malignomrisiko erhöht ist und eine immunsuppressive Therapie dieses Risiko weiter steigern könnte, oder es werden Fälle von Lymphomen, Leukämien oder tödlich verlaufenden Malignomen erwähnt. Dies führt eher zur Verunsicherung von Arzt und Patient, als dass es eine Hilfe in der Entscheidungsfindung darstellt. In randomisierten placebokontrollierten Studien zur Untersuchung der Sicherheit von DMARDs sind aktuelle Tumorerkrankungen oder Malignome in der Vergangenheit nahezu ausnahmslos ein Ausschlusskriterien. Auch eignen sich Fallzahlen und Nachbeobachtungszeiten der Zulassungsstudien nicht, um relativ seltene Ereignisse mit langen Latenzen wie Malignome - zu erfassen. Helfen können hier Registerdaten, die auch Patienten mit Malignomanamnese beobachten. Doch sind bislang auch hier die Fallzahlen und Beobachtungszeiten unter den einzelnen zur Verfügung stehenden DMARDs meist noch zu gering, um statistisch signifikante Aussagen treffen zu können. Dennoch bilden Auswertungen von Registern eine Datenbasis, die uns im Alltag bei Therapieentscheidungen behilflich sein kann, zumal in den 


\begin{tabular}{|ll}
\hline Odds & $\begin{array}{l}\text { Zahl der Ereignisse dividiert durch die Zahl der Nicht-Ereignisse } \\
\text { Skala von } 0 \text { bis unendlich }\end{array}$ \\
\hline Risiko & $\begin{array}{l}\text { Zahl der Ereignisse dividiert durch die Gesamtzahl des zugrunde liegenden Kollektivs } \\
\text { Skala von } 0 \text { bis } 100 \% \text { oder von } 0 \text { bis 1 }\end{array}$ \\
\hline Hazard-Rate & Zahl der Ereignisse in einem bestimmten Zeitraum \\
\hline Odds Ratio (OR) & Quotient aus den Odds zweier Gruppen (z. B. Erkrankte/Nicht-Erkrankte) \\
\hline Relatives Risiko (RR) & Quotient aus den Risiken zweier Gruppen \\
\hline Hazard Ratio (HR) & Quotient aus den Hazard-Raten zweier Gruppen \\
\hline $\begin{array}{l}\text { Bei geringen Wahrscheinlichkeiten sind OR und RR etwa in der gleichen Größenordnung, und das OR ist immer kleiner als das RR. Bei hohen Wahr- } \\
\text { scheinlichkeiten liegen die OR-Zahlen erheblich höher als die RR-Zahlen } \\
\text { Die Hazard Ratio unterscheidet sich vom relativen Risiko insofern, als letztere Ratio (RR) kumulativ über eine gesamte Studienperiode errechnet wird, } \\
\text { wohingegen erstere (HR) das unmittelbare Risiko über den Studienzeitraum repräsentiert. Hazard Ratios neigen etwas weniger zu Selektionsbias in } \\
\text { Bezug auf die gewählten Endpunkte }\end{array}$ \\
\hline $\begin{array}{l}\text { "Standard incidence ratio“ } \\
\text { Quotient aus beobachteter und erwarteter Zahl von Erkrankungsfällen in einer Population. Die erwartete Zahl errech- } \\
\text { net sich aus der Gesamtzahl der Angehörigen einer Population und der Erkrankungsrate }\end{array}$ \\
\hline
\end{tabular}

nächsten Jahren die Resultate aussagekräftiger Analysen mit größeren Fallzahlen zu erwarten sind.

Einen Überblick über die wichtigsten Begriffe der Epidemiologie gibt • Tab. 1.

\section{Aussage in Leitlinien}

Mehrere rheumatologische Leitlinien zur Therapie v. a. der RA beschäftigen sich mit dem Thema der Malignomanamnese. Im Folgenden sollen diejenigen Arbeiten Erwähnung finden, die dies am ausführlichsten tun. Alle Leitlinien verweisen jedoch auf die schwierige Datenlage.

Die nationalen Leitlinien Frankreichs von 2011 beziehen sich nur auf die Therapie mit TNF-Inhibitoren und sprechen folgende Empfehlungen aus [1]:

- Bei soliden Malignomen außer

Hautkrebs, die sich noch nicht

lange in Remission befinden, sollte auf gar keinen Fall eine TNF- $a$ inhibitorische Therapie begonnen werden.

- Bei einer länger als 5 Jahre währenden Remission können TNF- $\alpha$ Inhibitoren erwogen werden.

- Dagegen sind sie kontraindiziert, wenn sich das Malignom zwar scheinbar in Remission befindet, aber die initiale onkologische Therapie nicht komplettiert werden konnte oder initial Lymphknoten- oder Fernmetastasen bestanden haben.

- Bei Malignomen mit hoher Heilungsrate, deren Nachbeobachtungszeit aber noch zu kurz ist, um eine Hei- lung festzustellen, können TNF- $\alpha$ Inhibitoren erwogen werden, z. B. wenn der Primärtumor sehr klein war, keine Invasion oder Metastasierung vorlag und eine R0-Resektion erfolgte. Ähnliche Überlegungen gelten bei transformierten Kolonpolypen oder Carcinomata in situ (z. B. der Schilddrüse oder der Prostata). Nicht nur patientenbezogene Faktoren, sondern auch Risikofaktoren aus der Familienanamnese sollten berücksichtigt werden.

- Bei Malignomen mit hohem Rezidivrisiko (z. B. Melanom oder Mammakarzinom) sollte selbst bei 10 Jahre bestehender Remission größere Vorsicht bei der Indikationsstellung herrschen.

Diese Leitlinie spricht wesentliche Punkte an, die bei der gemeinsamen Entscheidung mit dem Patienten berücksichtigt werden müssen, allen voran das durch die Tumorcharakteristika bei Erstdiagnose festgelegte individuelle Rezidivrisiko.

In den kanadischen Leitlinien von 2012 zur Therapie der RA mit Nicht-Biologika- und Biologika-DMARDs werden folgende Aussagen getroffen [2]:

- Bei Patienten mit unkontrollierter Tumorerkrankung sollte eine traditionelle Basistherapie oder eine Biologikatherapie bis zum Abschluss von Chemotherapie oder Radiotherapie verschoben werden. Eine individuelle Entscheidung nach Ab- sprache zwischen Rheumatologen und Onkologen wird gefordert.

- Bei zurückliegender Lymphomerkrankung können evtl. Hydroxychloroquin, Sulfasalazin und Rituximab verwendet werden. Eine Therapie mit TNF- $\alpha$-Inhibitoren wird nicht empfohlen. Andere traditionelle Basistherapeutika oder Biologika sollen nur mit Vorsicht eingesetzt werden.

- Bei zurückliegendem Nicht-Melanom-Hautkrebs können evtl. traditionelle Basistherapeutika verwendet werden. Biologika dagegen sollen nur mit Vorsicht eingesetzt werden.

- Bei zurückliegendem solidem Malignom können evtl. traditionelle Basistherapeutika verwendet werden. Biologika dagegen sollen nur mit Vorsicht eingesetzt werden.

Die Leitlinien des American College of Rheumatology (ACR) äußern sich in ihrer Aktualisierung von 2012 noch dezidierter [3]:

- Für Patienten, deren onkologische Therapie für ein solides Malignom oder einen Nicht-Melanom-Hautkrebs mehr als 5 Jahre zurückliegt, sieht das Expertengremium keine Einschränkung in Bezug auf eine Biologikatherapie.

- Für Patienten, deren onkologische Therapie für ein solides Malignom oder einen Nicht-Melanom-Hautkrebs weniger als 5 Jahre zurückliegt oder die jemals ein malignes Lymphom hatten, hält das Expertengre- 
mium unter den Biologika lediglich Rituximab für ausreichend sicher.

In der Veröffentlichung der Leitlinien wird letztere Empfehlung einschränkend kommentiert und betont, dass sich die Evidenz für Rituximab v. a. von Populationen ohne RA mit Malignomanamnese herleitet.

Andere Leitlinien zur Differenzialtherapie der RA, z.B. die deutsche oder die europäische, sind zum Thema Malignomanamnese weniger spezifisch und mahnen zu Vorsicht sowie zu entsprechender Aufklärung, ohne dezidierte Handlungsanweisungen $\mathrm{zu}$ geben.

\section{Direkte Evidenz zum Rezidivrisiko}

Bislang haben nur wenige Studien die Therapie mit DMARDs bei Patienten mit Malignomanamnese primär untersucht. Im Folgenden sollen die wichtigsten Arbeiten zu dieser Fragestellung dargestellt werden.

In einer 2005 veröffentlichten Publikation werden Ergebnisse des prospektiven CORRONA (Consortium of Rheumatology Researchers of North America)-Registers vorgestellt [4]. Von den 9123 eingeschlossenen Patienten mit RA hatten 594 Patienten maligne Vorerkrankungen. Die Autoren konnten keine erhöhte Rate inzidenter oder rezidivierter Tumoren bei Patienten unter TNF-InhibitorTherapie im Vergleich zur traditionellen Basistherapie feststellen.

Dixon et al. [5] identifizierten im Biologikaregister der Britischen Gesellschaft für Rheumatologie (BSRBR) aus mehr als 14.000 Patienten 293 mit einem zurückliegenden Malignom. Patienten mit einem Nicht-Melanom-Hautkrebs wurden dabei ausgeschlossen; 177 dieser Patienten erhielten im weiteren Verlauf nach Entscheidung des behandelnden Arztes TNF-Inhibitoren (TNFi), 117 erhielten Nicht-Biologika-DMARDs (nbDMARDs). Numerisch war die Inzidenz von Malignomen in der Gruppe unter TNFi sogar geringer (25,3/1000 Patientenjahre [TNFi] vs. 38,3 [nbDMARDs]), wenn auch statistisch nicht signifikant (alters- und geschlechtsadjustiertes Inzidenzratenverhältnis von 0,58 [95\%-KI

Z Rheumatol 2016 · 75:22-31 DOI 10.1007/s00393-015-0034-6

(c) Springer-Verlag Berlin Heidelberg 2015

M. Schmalzing · A. Strangfeld · H.-P. Tony

Medikamentöse Therapie der rheumatoiden Arthritis bei Malignomanamnese. Epidemiologische Daten

\section{Zusammenfassung}

Hintergrund. Bei der Frage, ob eine immunsuppressive oder eine Biologikatherapie bei Malignomanamnese möglich und sicher ist, kann man sich nur auf eine begrenzte Datenlage berufen.

Methodik. Es erfolgte eine Recherche in PubMed, EULAR-Abstracts sowie ACRAbstracts 2013 bis 2015.

Ergebnisse. Die französische sowie die kanadische Fachgesellschaft und das American College of Rheumatology unternehmen den Versuch, konkrete Empfehlungen zu dieser Problematik zu geben. Direkte Evidenz bezieht sich v. a. auf Daten aus 3 nationalen Registern, die den Schluss nahelegen, dass eine Therapie mit TNFInhibitoren oder Rituximab bei der üblichen vorsichtigen Vorauswahl der Patienten mit Malignomanamnese zumindest bei längerem Abstand zur Malignomtherapie möglich erscheint. Für sämtliche gängigen Medikamente der medikamentösen Therapie der rheumatoiden Arthritis scheint kein eindeutig erhöhtes De-novo-Malignomrisiko vorzuliegen bei teils widersprüchlichen Ergebnissen. Die aktuelle Datenlage zu den einzelnen Substanzen wird dargestellt. Schlussfolgerung. Vor dem Hintergrund der aktuellen Literatur wird der Versuch eines Therapiealgorithmus zur medikamentösen Therapie der rheumatoiden Arthritis bei Malignomanamnese unternommen.

\section{Schlüsselwörter}

Biologika · Register · Immunsuppression . TNF-Inhibitoren · Rituximab

\section{Medication treatment of rheumatoid arthritis with a history of malignancy. Epidemiological data}

\section{Abstract}

Introduction. Only insufficient data are available regarding the question whether treatment with immunosuppressants or biologicals is feasible and safe in patients with a history of malignancy.

Method. Literature search via PubMed, EULAR abstracts and ACR abstracts from 2013 to 2015.

Results. The Société Francaise de Rhumatologie, the Canadian Rheumatology Association and the American College of Rheumatology have tried to make recommendations on this topic. Direct evidence mainly originates from data in three national registries which suggest that treatment with tumor necrosis factor (TNF) inhibitors and rituximab appears to be safe for carefully selected patients, at least if there is a longer interval between treatment with biologicals and oncological treatment. Furthermore, despite partly conflicting data all routine drugs for treating rheumatoid arthritis do not seem to show a consistently increased risk of de novo malignancies. The currently available data are presented for each drug of interest.

Conclusion. Taking the current literature into account an attempt is made to formulate an algorithm for the medicinal treatment of patients with rheumatoid arthritis and a history of malignancy.

\section{Keywords}

Biologics · Registries - Immunosuppression . TNF inhibitors · Rituximab
0,23-1,43]). Nur 1 Patient entwickelte ein Lokalrezidiv, alle anderen Tumoren stellten Erstmanifestationen dar. Als Erklärung für die im Vergleich mit nbDMARDs niedrigere Inzidenzrate unter TNFi vermuteten die Autoren, dass Patienten mit malignen Vorerkrankungen eher dann für eine Biologikatherapie ausgewählt wurden, wenn die Wahrscheinlichkeit für ein Tumorrezidiv von den behandelnden Ärzten als gering eingestuft wurde oder wenn der Tumor schon sehr lange in Remission war. Sie schlussfolgerten, dass bei dieser Selektion der Patienten kein erhöhtes Risiko unter TNF-Inhibitoren festzustellen ist, dass aber daraus keineswegs geschlossen werden kann, dass TNF-Inhibitoren bei Patienten mit Tumoranamnese auf alle Fälle sicher anzuwenden sind. 
Auch die aktualisierte Auswertung des Registers für den EULAR-Kongress 2014 mit 425 Patienten mit Malignomanamnese (nbDMARD-Therapie: 159, TNF-I: 243, Rituximab: 23) zeigte eine geringere Rate an Rezidiven unter TNFi (24/1000 Patientenjahre) und Rituximab (25/1000 Patientenjahre) im Vergleich zu nbDMARDs (47/1000 Patientenjahre) [6]. Allerdings war die Zeitdauer zwischen Tumor und Beginn der Therapie ziemlich lange und betrug im Median bei nbDMARDs 7,9 Jahre und bei TNFiTherapie sogar 11,5 Jahre. Bis zu einem Therapiebeginn mit Rituximab waren im Median nur 5,4 Jahre seit dem Tumor vergangen. Die alters- und geschlechtsadjustierten Hazard-Raten betrugen im Vergleich zur Therapie mit nbDMARDs für TNFi 0,55 (95\%-KI 0,35-0,87) und für Rituximab 0,47 (95\%-KI 0,11-1,94). Trotz der von den Autoren als zu gering eingeschätzten Fallzahlen für ein signifikantes Ergebnis deuten die Daten doch darauf hin, dass die in Großbritannien mit Biologika therapierten RA-Patienten mit Tumoranamnese kein erhöhtes Risiko für ein Rezidiv haben im Vergleich $\mathrm{zu}$ denjenigen, die mit nbDMARDs behandelt werden.

Im deutschen RABBIT (Rheumatoide Arthritis - Beobachtung der Biologika Therapie)-Register hatten $122 \mathrm{der}$ 5120 Patienten mit RA eine Malignomanamnese [7]; 58 dieser Patienten erhielten TNFi, 9 Anakinra und 55 nbDMARDs. Verglichen mit Patienten unter nbDMARDs (31,4/1000 Patientenjahre) hatten Patienten unter TNFi keine signifikant höhere Rate an Tumorrezidiven (45,5/1000 Patientenjahre) mit einem Inzidenzratenverhältnis von $1,4(\mathrm{p}=0,6)$. Die mediane Zeit zwischen der malignen Vorerkrankung und Beginn der Therapie war im Gegensatz zu den im britischen Register beobachteten Patienten deutlich kürzer und betrug für beide Gruppen etwa 5 Jahre. Anders als in der britischen Studie wiesen 14 von 15 Tumorrezidiven die gleiche Histologie wie der Primärtumor auf. Die mediane Zeit zwischen dem primären Tumorgeschehen und der Diagnose eines neuen Tumors unter Therapie betrug für Patienten unter TNF- $\alpha$ Inhibitoren 9,5 Jahre und für Patienten unter konventionellen Basistherapeutika
9,2 Jahre. Bei 3 Patienten trat ein Tumorrezidiv innerhalb von 5 Jahren nach Erstdiagnose des primären Malignoms auf. Zwei Patienten davon hatten TNFi erhalten.

In der Aktualisierung der RABBIT-Daten von 2013 hatten 367 von 10.168 RA-Patienten (3,6\%) eine Malignomanamnese [8]. Ein relativ hoher Anteil von $28 \%$ erhielt Rituximab. Die durchschnittlichen Nachbeobachtungszeiten unter Biologikatherapie waren mit 3 Jahren bei stattgehabtem Lymphom und mit 2,6 Jahren bei stattgehabtem solidem Malignom noch relativ kurz. Dafür fiel auf, dass Biologikatherapien deutlich zeitiger nach Malignomanamnese verschrieben worden waren, als aus den anderen Registern berichtet: Bei TNFInhibitoren lagen nur 6,8 Jahre, bei Rituximab sogar nur 3,3 Jahre zwischen Malignom und Therapiebeginn. Die Rezidivraten unterschieden sich nicht wesentlich und betrugen im Fall von soliden Malignomen (in 1/100 Patientenjahren) 3,6 für nbDMARDs, 3,9 für Rituximab und 5,7 für TNFi. Es scheint, als ob diese Daten das in den amerikanischen Leitlinien aufgrund des Wirkmechanismus und der Extrapolation von anderen Patientengruppen vermutete geringe Rezidivrisiko unter Rituximab stützen könnten.

\section{I) Es besteht vermutlich ein geringes Rezidivrisiko unter Rituximab}

Der Vergleich der Ergebnisse des britischen Registers mit denen des RABBITRegisters legt nahe, dass die Zeitdauer zwischen Tumorerkrankung und Therapiebeginn mit TNFi eine Rolle spielen könnte. Dieser Aspekt sowie die tumorspezifische Rezidivwahrscheinlichkeit müssen zukünftig noch besser untersucht werden.

Durch Verlinkung der Daten des nationalen schwedischen Krebsregisters mit dem Register ambulant behandelter (Biologika-naiver) RA-Patienten und denen des schwedischen Biologikaregisters (ARTIS) wurde das Rezidivrisiko unter TNFi nach Mammakarzinom untersucht [9]. Diese Fragestellung ist von besonderem Interesse, weil gerade das Mammakarzinom auch nach vielen Jahren rezidivieren kann und es das häufigste Malignom in der weiblichen RA-Population darstellt. Die Untersuchung war als Fall-Kontroll-Studie angelegt, in der nach Geschlecht, Alter bei Tumorerkrankung, Kalenderjahr bei Erstdiagnose (ED) des Mammakarzinoms und Tumorstadium bei ED (invasiv/in situ) sowie Wohnregion gematcht wurde. Unter 143 Patientinnen mit TNFi-Therapie und 1598 Biologika-naiven Patientinnen konnten 120 Paare identifiziert werden, bei denen beide Partner die Matchingkriterien erfüllten. Die mittlere Beobachtungszeit unter Therapie betrug mehr als 4,5 Jahre. Als weiteres Kriterium für den Einschluss in die Analyse wurde festgelegt, dass die Tumorerkrankung bei Therapiebeginn in Remission sein musste. Über die im Krebsregister gespeicherten Informationen konnten zusätzlich weitere Tumorcharakteristika erhoben werden (wie Hormonrezeptorstatus, Lymphknotenbefall, Grading, brusterhaltende Operation oder Mastektomie sowie adjuvante Chemotherapie). Die rohen Rekurrenzraten betrugen 16 (7-31)/1000 Patientenjahre bei Biologika-naiven Patientinnen und 15 (7-29)/1000 Patientenjahre unter TNFi-Therapie. Adjustiert für die etwas schlechtere Ausgangssituation der nbDMARD-Patientinnen (z. B. $28 \% \mathrm{mit}$ positivem Lymphknotenbefall im Gegensatz $\mathrm{zu} 15 \%$ in der TNFi-Gruppe) und deren dadurch etwas schlechtere Prognose zeigte sich unter TNFi im Vergleich zu nbDMARDs kein erhöhtes Risiko für eine Rekurrenz (HazardRate 1,1 [0,4-2,8]). Dieses insgesamt sehr positive Ergebnis wird nur dadurch eingeschränkt, dass erneut keine Aussage getroffen werden kann, ob eine nach Malignomerkrankung frühzeitig begonnene TNFi-Therapie das Rekurrenzrisiko erhöht, denn auch in dieser Untersuchung war der Zeitraum zwischen Tumor und Therapiebeginn mit TNFi mit im Median 9,4 Jahren recht hoch.

Eine weitere, sich auf eine Tumorentität bzw. in diesem Fall eine spezifische Präkanzerose beschränkende Untersuchung wurde vom britischen Register 
veröffentlicht [10]. Von 11.738 im Register eingeschlossenen Patientinnen konnten durch die Verlinkung mit dem nationalen britischen Krebsregister 238 Patientinnen identifiziert werden, bei denen eine CIN III (zervikale intraepitheliale Neoplasie Stadium III), eine obligate Präkanzerose, an das Krebsregister gemeldet worden war. In der nbDMARD-Kontrollgruppe wurden 48 der Patientinnen mit anamnestischer CIN III beobachtet, die verbleibenden 190 hatten bei Einschluss in das Biologikaregister eine TNFi-Therapie begonnen. Als Endpunkte wurden das Auftreten von Krebserkrankungen des weiblichen Genitaltraktes, Tod oder Ende der Beobachtung definiert. Nach einer medianen Beobachtungszeit von 3,9 (nbDMARDs) bzw. 5,2 (TNFi) Jahren im Register und einem medianen Abstand von 14 (nbDMARDs) bzw. 13 (TNFi) Jahren zwischen registrierter CIN III und Therapiebeginn waren in der Gruppe der nbDMARD-behandelten Frauen 2 neue Malignome des Genitaltraktes aufgetreten (beide mit tödlichem Ausgang), während in der Gruppe unter TNFi-Therapie keine neuen Malignome gemeldet worden waren. Dies sind bislang die einzigen Daten, die es zur Therapie mit Biologika nach obligater Präkanzerose gibt. Wieder ist der sehr lange Zeitraum zwischen Präkanzerose und Therapiebeginn auffällig (nur 14\% der TNFi-therapierten Frauen begannen die Therapie innerhalb der ersten 5 Jahre nach CIN III), allerdings hatten in dieser Untersuchung auch viele Patientinnen erst einige Jahre nach der Präkanzerose die rheumatische Erkrankung entwickelt.

\section{Daten zu einzelnen Medikamenten}

Im Folgenden sollen Daten zum Malignomrisiko für Medikamente zur Therapie der RA dargestellt werden. Dabei handelt es sich fast ausschließlich um Untersuchungen zur Inzidenz von De-novoMalignomen und nicht um Daten zum Tumorrezidivrisiko. Bei fehlender direkter Evidenz bleibt allerdings nichts anderes übrig, als zu postulieren, dass diese Risiken miteinander verknüpft sind. Und so würde es zumindest beim Einsatz von DMARDs bei Malignomanamnese eine gewisse Beruhigung bedeuten, wenn für das betreffende Präparat bei guter Datenlage kein vermehrtes Auftreten neuer Tumorerkrankungen bekannt ist.

\section{Glukokortikosteroide}

In manchen Studien waren Dosis und Dauer der Glukokortikoidtherapie mit einem höheren Risiko für Basaliome, Plattenepithelkarzinome, Non-Hodgkin-Lymphome und maligne Melanome assoziiert. Die Kausalität dieses erhöhten Malignomrisikos wurde dagegen von anderen Studien infrage gestellt. Die widersprüchliche Datenlage erlaubt vielleicht den Schluss, dass der Effekt von Glukokortikosteroiden auf das Malignomrisiko eher gering sein dürfte, v. a. wenn niedrige Dosierungen oder eine kurze Behandlungsdauer gewählt werden [11].

\section{Nicht-Biologika-DMARDs}

\section{Methotrexat}

Schon länger sind Sammlungen von Lymphomfällen oder lymphoproliferativen Erkrankungen unklarer Dignität unter Methotrexat (MTX)-Therapie bekannt. Suggestiv für einen kausalen Zusammenhang war dabei auch die Tatsache, dass sich diese Lymphome in einzelnen Fällen nach Absetzen von MTX zurückgebildet hatten.

Die kanadischen Leitlinien verweisen zu diesem Thema auf eine Fall-KontrollStudie, die das Risiko neuer Lymphome von RA-Patienten unter MTX mit dem unter anderen Nicht-BiologikaDMARDs vergleicht [12]. Es wurde lediglich ein Trend zu einem höheren Risiko gefunden, der sich nicht als statistisch signifikant herausstellte (RR 1,23, $95 \%$-KI 0,97-1,57).

In der Arbeit von Wolfe et al. von 2004 wurden 18.572 RA-Patienten prospektiv in Bezug auf das Lymphomrisiko ausgewertet und nicht nur der Effekt von MTX, sondern auch von TNF- $\alpha$-Inhibitoren untersucht [13]. Numerisch lag die standard incidence ratio (SIR) für das Auftreten eines neuen Lymphoms unter MTX höher als unter anderen NichtBiologika-DMARDs. Dieser Unterschied war allerdings nicht statistisch signifi- kant. Das leicht erhöhte Risiko in der TNF-Inhibitor-Gruppe wurde dadurch erklärt, dass es sich um Patienten mit höherer Krankheitsaktivität und damit mit einem per se höheren Lymphomrisiko gehandelt hatte.

Die Untersuchung von Buchbinder et al. [14] in Australien überblickt 4145 Personenjahre MTX-Therapie bei RA und kommt zu dem Schluss, dass diese Patienten zumindest im Vergleich zur Normalbevölkerung ein höheres Risiko für maligne Melanome, NonHodgkin-Lymphome und Lungenkarzinome aufweisen. Der Vergleich zu RAPatienten ohne MTX-Therapie bzw. mit einer alternativen Basistherapie konnte nicht getroffen werden.

Aus der Analyse der Medicare-Datenbank für den ACR-Kongress 2014 geht hervor, dass eine MTX-Therapie bei einer Anwendungszeit von über $1 \mathrm{Jahr}$ das Risiko für Nicht-Melanom-Hautkrebs leicht, aber signifikant erhöht [15]. Dies galt in der gleichen Studie nicht für TNF-I, Abatacept oder Rituximab.

Letztlich sei noch eine aktuelle Auswertung des CORRONA-Registers erwähnt, in der 6806 Basistherapieverläufe ausgewertet wurden [16]. Das Risiko für eine inzidente Krebserkrankung war höher unter MTX im Vergleich zu anderen Nicht-Biologika-Basistherapeutika und im Vergleich zu TNF-Inhibitoren. Die Krebsrate unter Abatacept oder Rituximab dagegen unterschied sich nicht signifikant von der unter MTX. Die Autoren betonen aber, dass ein nicht kontrolliertes Studiendesign vorliegt und die unterschiedlichen Raten durch Vorselektion der rekrutierenden Ärzte bedingt sein könnten.

\section{》) MTX hat keinen deutlichen Effekt auf das Malignomrisiko}

Trotz der widersprüchlichen Ergebnisse kann man aufgrund der langjährigen Erfahrung mit hohen Fallzahlen in Studien und Registern davon ausgehen, dass MTX keinen deutlichen Effekt auf das Malignomrisiko hat. 


\section{Leflunomid, Sulfasalazin, Hydroxychloroquin}

Sulfasalazin und Hydroxychloroquin werden in den kanadischen Leitlinien bei Malignomanamnese als unbedenklich angesehen, wobei es sich hierbei um eine Expertenmeinung handelt. Für Sulfasalazin wurde sogar ein protektiver Effekt gegen Malignomentstehung bei Colitis ulcerosa angenommen, und in vitro wurden wachstumshemmende Effekte bei Tumorzelllinien gesehen. In vivo konnte dagegen kein derartiger Effekt gezeigt werden. Das karzinogene Potenzial von Hydroxychloroquin wurde in einer Langzeitstudie an Ratten erprobt, die keinen nachteiligen Effekt zeigen konnte. In einer nicht randomisierten Studie mit 235 Patienten mit systemischem Lupus erythematodes hatten Patienten, die jemals ein Antimalariamittel erhielten, ein signifikant niedrigeres Malignomrisiko als Patienten, die niemals diese Medikamente einnahmen [17].

Auch für Leflunomid existieren aus tierexperimentellen Daten keine Hinweise auf ein erhöhtes Malignomrisiko. In einer Übersichtsarbeit zur LeflunomidTherapie bei RA weisen Behrens et al. [18] aber auf 7 Fälle von Pankreaskarzinomen hin, die unter oder nach Leflunomid auftraten und im deutschen RABBITRegister erfasst wurden. Dieser Cluster hätte eine Verdreifachung des Risikos eines Pankreaskarzinoms im Vergleich zur Normalbevölkerung bedeutet. Dieser Zusammenhang bestätigte sich aber nicht im britischen oder schwedischen Biologikaregister. Darüber hinaus konnten für Metaboliten von Leflunomid in vitro sogar Antitumoreffekte bei MultipleMyelom- oder Prostatakarzinom-Zelllinien gezeigt werden.

\section{Ciclosporin A}

Wenn man Daten aus dem Transplantationsbereich extrapoliert, scheint beim Einsatz von Ciclosporin A (CSA) bei Malignomanamnese zumindest in Kombination mit Glukokortikosteroiden und anderen Immunsuppressiva Vorsicht geboten, wobei der Effekt von CSA selbst nicht genau benannt werden kann. Von dermatologischer Seite wird häufig vor dem Einsatz von CSA aufgrund des Hautkrebsrisikos gewarnt. Muellenhof et al.
[19] werteten aber in ihrer Metaanalyse 60 Studien mit über 1700 Patienten aus und kamen zu dem Ergebnis, dass CSA das Hautkrebsrisiko wahrscheinlich nicht substanziell erhöht, sofern dermatologische Leitlinien beachtet werden (niedrige Dosis mit maximal $5 \mathrm{mg} / \mathrm{kg}$ pro Tag, keine Kombination mit anderen Immunsuppressiva, keine sonstigen Risikofaktoren für Karzinogenese wie stattgehabter Hautkrebs, zeitlich begrenzter Einsatz von maximal 2 Jahren etc.).

\section{Biologika-DMARDs}

\section{TNF-Inhibitoren}

Die ersten TNF-Inhibitoren (TNFi) wurden 2001 zugelassen und werden seitdem zur Therapie von entzündlich rheumatischen Systemerkrankungen eingesetzt. Seit deren Zulassung wurden in verschiedenen europäischen Ländern Biologikaregister initiiert mit dem Ziel, die Langzeitsicherheit und -wirksamkeit dieser neuartigen - weil zytokingerichteten - Therapien zu untersuchen. Besonderes Augenmerk wurde auf ein durch den Wirkmechanismus vermutetes erhöhtes Malignomrisiko gelegt. Durch diese Langzeitkohorten ist die Datenlage für TNFi nach fast 15 Jahren Anwendungserfahrung besser als für alle anderen DMARDs.

Fallberichte von Patienten mit chronisch entzündlichen Darmerkrankungen weisen auf ein erhöhtes Risiko für T-ZellLymphome unter TNFi hin. Die ausführliche Auswertung aus der FDA-Datenbank von Deepak et al. konnte lediglich bei Komedikation mit Thiopurinen ein gering erhöhtes Risiko für diese Lymphomentität im Vergleich zu Patienten unter 5-Aminosalicylat-Therapie zeigen [20].

Eine Analyse des französischen RATIO-Registers bezüglich des Risikos inzidenter Lymphome unter Infliximab, Adalimumab und Etanercept [21] zeigte eine insgesamt erhöhte Lymphomrate in der Kohorte im Vergleich zur Normalbevölkerung, lag jedoch laut den Autoren in dem Bereich, der für RA-Patienten zu erwarten wäre.

Mittlerweile liegen mehrere Metaanalysen von Registerdaten, von Daten aus Anwendungsbeobachtungen und von Daten aus randomisierten Studien vor, die kein erhöhtes Malignomrisiko auch in Bezug auf andere Entitäten identifizieren konnten [22-30].

Die größte und derzeit aktuellste Analyse stammt aus dem britischen Register mit 11.767 TNFi-behandelten Patienten (52.549 Patientenjahre Beobachtung) und 3249 nbDMARD-behandelten Patienten (11.672 Patientenjahre Beobachtung). Die in dieser Untersuchung zugrunde liegenden Registerdaten konnten mit denen des nationalen britischen Krebsregisters verbunden werden. Insgesamt waren 563 solide Tumoren aufgetreten. Durch die Größe der zugrunde liegenden Datenmenge hatte die Analyse ausreichend statistische Power, um das Risiko für solide Tumore auch für die einzelnen TNFi zu berechnen. Im Vergleich zu nbDMARD-behandelten Patienten betrugen die „propensity score"-adjustierten Hazard-Raten für Etanercept (0,9 [0,7-1,2]), Infliximab $(0,8[0,6-1,1])$ und Adalimumab $(0,8$ [0,6-1,1]) [22].

Bezüglich der Frage, ob sich der langjährige Einsatz von TNF-Inhibitoren bei Kindern, Jugendlichen und jungen Erwachsenen ungünstig auswirken könnte, ist eine aktuelle US-amerikanische Arbeit relevant, die junge Patienten (<30 Jahre) unter Etanercept-Therapie im Rahmen einer Anwendungsbeobachtungsstudie untersucht hat [31]. Es wurden 231.404 Patientenjahre analysiert. Lediglich für Hodgkin-Lymphome in der Altersgruppe 0 bis 17 Jahre wurde eine signifikant höhere Rate im Vergleich zur Normalbevölkerung gefunden. Die Autoren betonen aber, dass kein Vergleich zu einer Patientengruppe ohne Etanercept-Therapie gezogen wurde und dass die rheumatisch entzündliche Erkrankung per se das Lymphomrisiko erhöht. Im deutschen Register „Biologika in der Kinderrheumatologie" wurde allerdings keine erhöhte Malignomrate bei 1414 Patienten mit juveniler idiopathischer Arthritis unter Etanercept- oder Adalimumab-Therapie im Vergleich zu Patienten unter MTX-Therapie gefunden [32].

Insgesamt zeigen die Daten verschiedener europäischer Register bislang keine Erhöhung des Malignomrisikos unter TNFi-Exposition im Vergleich zu nbD- 
MARDs. Dies ist das Ergebnis einer Metaanalyse von 9 Publikationen aus Biologikaregistern, in denen das Malignomrisiko untersucht wurde [33].

Inwieweit TNF-Inhibitoren das Risiko für unterschiedliche Hautkrebsentitäten beeinflussen, wird ausführlich in dem Artikel von Burkhardt et al. in dieser Ausgabe behandelt.

\section{Rituximab}

Rituximab nimmt zumindest in den USamerikanischen Leitlinien eine herausgehobene Stellung bei der Therapie von Patienten mit malignen Vorerkrankungen ein. Für Patienten mit stattgehabten B-Zell-Lymphomen, für die das Präparat teilweise als Erhaltungstherapie eingesetzt werden kann, erschließt sich die Empfehlung unmittelbar. Die Leitlinie beruft sich auf Literatur zum Einsatz von Rituximab bei Nicht-RA-Indikationen, die aber nicht weiter spezifiziert wird.

Für Patienten mit RA kann man sich v. a. auf die Arbeit von van Vollenhoven et al. berufen, die sog. „ungünstige Ereignisse von Interesse" unter RituximabTherapie über einen Nachbeobachtungszeitraum von 9,5 Jahren im gesamten Studienprogramm von Rituximab auswertet [34]. Im Vergleich zu RA-Patienten ohne Rituximab-Vortherapie oder zur Normalbevölkerung zeigten sich keine erhöhten Raten von Malignomen. Dies galt auch für Patienten, die mehrfach eine Retherapie erhielten. Diese Analyse wurde nochmals nach 14.816 Patientenjahren für den EULAR-Kongress 2013 aktualisiert und erbrachte das gleiche beruhigende Ergebnis.

Letztlich fiel auch die aktuellste Analyse des deutschen RABBIT-Registers zum Malignomrezidivrisiko wie oben erwähnt zugunsten von Rituximab aus.

\section{Tocilizumab}

Tocilizumab selbst wurde gezielt zur Therapie von Neoplasien wie dem Morbus Castleman eingesetzt. Ebenso weisen Einzelfallberichte auf günstige Effekte bei Plasmazelldyskrasien hin [35].

Die erste größere Übersichtsarbeit zur Sicherheit von Tocilizumab in Bezug auf das Risiko neuer Malignome stammt aus Japan und wurde 2012 veröffentlicht [36]. Obwohl die SIR für neue Malignome mit 1,1 pro 100 Patientenjahre unter einer Tocilizumab-Therapie von im Median 3,6 Jahren Dauer ungefähr den erwarteten Wert für RA-Patienten hatte, kommen die Autoren zu dem Schluss, dass Tocilizumab bei Patienten mit bestehenden Malignomen aufgrund der insuffizienten Datenlage nicht verwendet werden sollte.

In der neueren Arbeit von Genovese et al. zu Tocilizumab wurde kein erhöhtes Malignomrisiko im Vergleich zur Allgemeinbevölkerung gefunden [37]. Diese Arbeit wurde für den EULAR-Kongress 2013 noch aktualisiert und fand keine neuen Sicherheitssignale nach 16.204,8 Patientenjahren.

\section{Abatacept}

In der Analyse des gesamten Studienprogramms mit Beobachtungszeiträumen von bis zu 8 Jahren kamen Weinblatt et al. zu dem Ergebnis, dass sich zwar für manche Tumorentitäten tendenziell höhere (z. B. Lymphome und Bronchialkarzinome) oder niedrigere (z. B. Kolonkarzinome und Mammakarzinome) SIR-Werte zeigten, insgesamt unterschied sich die SIR nicht von der Normalbevölkerung [38]. Aktualisierungen dieser Daten für den ACR-Kongress 2012 und den EULAR-Kongress 2013 mit jeweils 16.670,56 Patientenjahren kamen zum gleichen Ergebnis. Für den EULAR-Kongress 2015 wurde speziell das Hautkrebsrisiko im gesamten Studienprogramm untersucht mit einer individuellen Exposition von durchschnittlich mehr als 30 Monaten [39]. Dieses Risiko war im Vergleich zur Placebogruppe nicht erhöht und im Verlauf stabil.

Gemäß einer Analyse des französischen ORA-Registers scheint sich bei Patienten mit monoklonaler Gammopathie unklarer Signifikanz $(\mathrm{n}=38)$ eine Abatacept-Therapie nicht ungünstig auszuwirken im Sinne eines Anstiegs des Paraproteins [40].

\section{Anakinra}

Der Interleukin-1-Rezeptor-Antagonist Anakinra wird zwar zur Therapie der RA nur noch selten eingesetzt, Daten zum Malignomrisiko stammen aber aus einer Übersichtsarbeit, in der bei RA-
Patienten 63 randomisierte Studien zu unterschiedlichen Biologika und im Fall von Anakinra 2 Studien mit insgesamt 2791 Patienten ausgewertet wurden [41]. Anakinra wies in Kombination mit MTX sogar ein niedrigeres relatives Risiko für Malignome im Vergleich zu Patienten auf, die nur MTX erhielten. Diese Daten sollten aber aufgrund der kleinen Fallzahlen und des kurzen Beobachtungszeitraumes mit Vorsicht interpretiert werden.

\section{Schlussfolgerung und Therapiestrategie}

Bei Empfehlungen zur DMARD-Therapie bei Anamnese eines soliden Malignoms kann man sich bislang nur auf eine begrenzte Datenlage berufen. Nur wenige Studien liefern aussagekräftige direkte Evidenz zu dieser Fragestellung. Daten zu Tumorrezidiven aus randomisierten placebokontrollierten Studien liegen nicht vor, da eine Malignomanamnese bis auf wenige Ausnahmen ein Ausschlusskriterium für derartige Studien darstellt. Die einzigen direkten Daten zu dieser Fragestellung, die zurzeit zur Verfügung stehen, stammen aus Registern. Deren größte Limitation wird jedoch durch die Selektion der Patienten zu den einzelnen Therapien (durch die Therapieentscheidung des behandelnden Rheumatologen) verursacht. Mit längerer Anwendungserfahrung und größeren Datenmengen in den Registern steigen jedoch die Chancen, robuste Untersuchungen zum Rezidivrisiko durchführen zu können. Gute Ergebnisse hierzu dürfen für die nächsten Jahre erwartet werden. Zu hoffen ist, dass auch die Frage geklärt werden kann, wie zeitig eine Biologikatherapie nach Malignomanamnese begonnen werden kann.

Bis dahin kann man versuchen aus den Daten zur De-novo-Malignomentstehung Rückschlüsse zu ziehen. Die Daten zu Cyclophosphamid, die nicht Gegenstand dieser Arbeit waren, sind diesbezüglich eindeutig ungünstig. Dagegen gilt für die anderen medikamentösen Therapeutika in der Rheumatologie und insbesondere für die Medikamente zur Therapie der RA, dass selbst bei uneinheitlichen Ergebnissen der Effekt auf die Neuentstehung und das Rezidivrisi- 
ko von Malignomen - wenn überhaupt

- nur gering sein dürfte.

Vor dem Hintergrund des bisher Gesagten scheint folgendes Vorgehen bei der Entscheidung über eine DMARD-Therapie bei Malignomanamnese möglich:

1. Zunächst gilt es zu klären, ob sich der Patient bezüglich seines Malignoms in Remission befindet. Falls nicht, würde sich die Frage anschließen, ob die rheumatisch entzündliche Erkrankung als Paraneoplasie bzw. als tumorassoziiert $\mathrm{zu}$ interpretieren ist. In diesem Fall könnte die onkologische Therapie auch die entzündliche Aktivität günstig beeinflussen und würde klare Priorität im Vergleich zur immunsuppressiven Therapie haben.

2. Anschließend sollten in enger Absprache mit dem onkologischen Kollegen im Falle einer Remission eine Analyse des Rezidivrisikos und im Falle einer aktiven malignen Erkrankung die Festlegung des Therapieziels erfolgen. Bei Tumorremission wird in den US-amerikanischen und den französischen Therapieempfehlungen ein Abstand von 5 Jahren zur kurativen onkologischen Therapie als relativ sicher für jegliche immunsuppressive Therapie eingeschätzt. Bei Malignomen mit hohem Rezidivrisiko (z. B. malignes Melanom) bzw. der Möglichkeit von späten Rezidiven (z. B. Mammakarzinom) werden größere Intervalle zwischen kurativer Tumortherapie und immunsuppressiver bzw. Biologikatherapie von den französischen Leitlinien empfohlen, ohne dass für diese Empfehlung zur damaligen Zeit belastbare epidemiologische Daten vorlagen. Dieses Vorgehen erscheint aber mittlerweile vor dem Hintergrund der Daten aus dem deutschen und dem britischen Biologikaregister gerechtfertigt.

3. Bei aktiver Tumorerkrankung steht bei palliativer Zielsetzung der onkologischen Therapie das Wohlbefinden des Patienten im Vordergrund. Eine wirksame immunsuppressive Therapie wäre daher in den meisten Fällen gut zu rechtfertigen, außer sie steht einer onkologischen Therapie mit höherer Priorität entgegen. Sollte da- gegen ein kuratives Konzept verfolgt werden, wäre die Indikation für ein DMARD strenger zu stellen.

4. Zur Therapie der RA bei Malignomanamnese sollten falls möglich, nichtmedikamentöse bzw. lokaltherapeutische Alternativen ausgeschöpft werden (lokale Glukokortikoidinstillationen, Radiosynoviorthesen oder operative Verfahren). Der Einsatz von nichtsteroidalen Antirheumatika (NSAR) und Glukokortikoiden wird in Bezug auf das Malignomrisiko als weitgehend unproblematisch angesehen.

5. Sofern eine DMARD-Therapie innerhalb von 5 Jahren nach kurativer onkologischer Therapie alternativlos erscheint, sollte ein Präparat mit möglichst günstiger Datenlage zum Malignomrisiko gewählt werden. Sulfasalazin und Hydroxychloroquin sind hierbei zu bevorzugen. Ansonsten gilt das oben zu den Einzelsubstanzen Gesagte.

6. In Situationen, in denen sich Patient und Arzt für eine Biologikatherapie nach kurativer Malignomtherapie entschieden hatten, wurde in den letzten Jahren vermehrt Rituximab eingesetzt, obwohl auch für dieses Medikament trotz günstiger Ergebnisse aus dem deutschen und dem britischen Biologikaregister keine sichere Datenlage besteht, die den unbedenklichen Einsatz insbesondere bei kurzer Latenz ( $<5$ Jahre) begründen würde.

7. Die Entscheidung über eine rheumatologische medikamentöse Therapie kann nur sehr individuell nach ausführlicher Aufklärung des Patienten über die eingeschränkte Datenlage zur Sicherheit bei Malignomanamnese getroffen werden. Dabei müssen sorgfältig Patientenwunsch, Leidensdruck und die Bedrohung durch die rheumatisch entzündliche Erkrankung auf der einen Seite und das Rezidivrisiko auf der anderen Seite abgewogen werden.

8. Patienten mit einem erhöhten Risiko für Neuentstehung bzw. Rezidiv eines Malignoms sollen konsequent und engmaschig Vorsorge- bzw.
Nachsorgeuntersuchungen erhalten.

Der beschriebene Therapiealgorithmus stützt sich auf den in der Arbeit von Elandt et al. [11] vorgeschlagenen sowie die genannten nationalen Leitlinien bzw. Therapieempfehlungen und wurde mit den Ergebnissen der nationalen Register in Beziehung gesetzt.

Abschließend sei nochmals darauf hingewiesen, dass die Entscheidung über eine DMARD-Therapie bei Malignomvorgeschichte nach ausführlichen Gesprächen zusammen mit dem Patienten und dem behandelnden Onkologen getroffen werden soll. Interdisziplinäre Zusammenarbeit und ein vertrauensvolles Arzt-Patienten-Verhältnis haben gerade bei dieser Fragestellung eine herausragende Bedeutung.

\section{Fazit für die Praxis}

- Bei der Frage, ob eine immunsuppressive oder eine Biologikatherapie bei Malignomanamnese möglich und sicher ist, kann man sich nur auf eine begrenzte Datenlage berufen.

- Eine Therapie mit TNF-Inhibitoren oder Rituximab scheint bei der üblichen vorsichtigen Vorauswahl der Patienten mit Malignomanamnese zumindest bei längerem Abstand zur Malignomtherapie möglich zu sein.

- Für alle gängigen Medikamente der medikamentösen Therapie der RA scheint kein eindeutig erhöhtes Denovo-Malignomrisiko vorzuliegen bei teils widersprüchlichen Ergebnissen.

- Die Entscheidung über eine DMARDTherapie bei Malignomvorgeschichte sollte zusammen mit dem Patienten und dem behandelnden Onkologen getroffen werden. 


\section{Korrespondenzadresse}

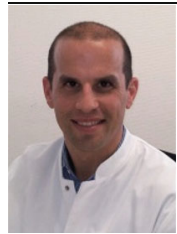

\section{Dr. M. Schmalzing}

Rheumatologie/klinische Immunologie, Medizinische Klinik und Poliklinik 2, Zentrum Innere Medizin, Universitätsklinikum Würzburg Oberdürrbacher Str. 6, 97080 Würzburg,

Deutschland schmalzing_m@ukw.de

\section{Einhaltung ethischer Richtlinien}

Interessenkonflikt. Dieses Manuskript entstand auf Basis einer von Roche und Chugai unterstützten Arbeitsgruppe (Systemerkrankung RA), aber ohne jegliche Einflussnahme der Firmen auf das Ergebnis oder die Publikation. M. Schmalzing hat Vortragsund/oder Beratungshonorare erhalten von AbbVie, Actelion, BMS, Chugai, Genzyme, Janssen-Cilag, Novartis, Pfizer, Roche, UCB. H.-P. Tony hat Vortrags- und/ oder Beratungshonorare erhalten von AbbVie, Chugai, Janssen-Cilag, MSD, Pfizer, Roche, UCB. A. Strangfeld hat Vortragshonorare erhalten von AbbVie, BMS, MSD, Pfizer, Roche, Sanofi-Aventis und UCB.

Dieser Beitrag beinhaltet keine Studien an Menschen oder Tieren.

\section{Literatur}

1. Pham T, Bachelez H, Berthelot JM et al (2011) TNF alpha antagonist therapy and safety monitoring. Joint Bone Spine 78(Suppl 1):15-185

2. Bombardier C, Hazlewood GS, Akhavan P et al (2012) Canadian rheumatology association recommendations for the pharmacological management of rheumatoid arthritis with traditional and biologic disease-modifying Antirheumatic drugs:part Il safety. J Rheumatol 39:1583-1602

3. Singh JA, Furst DE, Bharat A et al (2012) 2012 update of the 2008 american college of rheumatology recommendations for the use of disease-modifying Antirheumatic drugs and biologic agents in the treatment of rheumatoid arthritis. Arthritis Care Res 64(5):625-639

4. Maury EE et al (2005) Rheumatoid arthritis patients on tumor necrosis factor blockers do not have an increased risk of cancer. Arthritis Rheum 52(12):4055

5. Dixon WG, Watson KD, Lunt M, Mercer LK, Hyrich KL, Symmons DP (2010) Influence of anti-tumor necrosis factor therapy on cancer incidence in patients with rheumatoid arthritis who have had a prior malignancy: results from the British Society for Rheumatology Biologics Register. Arthritis Care Res (Hoboken) 62(6):755-763

6. Silva-Fernandez LL, Lunt M, Watson KD et al (2014) The influence of anti-TNF or Rituximab on cancer incidence in patients with rheumatoid arthritis who have had a prior malignancy. Ann Rheum Dis 73(Suppl2):674

7. Strangfeld A, Hierse F, Rau R, Burmester GR, Krummel-Lorenz B, Demary W, Listing J, Zink A
(2010) Risk of incident or recurrent malignancies among patients with rheumatoid arthritis exposed to biologic therapy in the German biologics register RABBIT. Arthritis Res Ther 12:R5

8. Strangfeld A, Pattloch D, Herzer P et al (2013) Risk of cancer recurrence or new tumors in RA patients with prior malignancies treated with various biologicagents. Arthritis Rheum 65(10):342

9. Raaschou P, Frisell T, Askling J (2014) TNF inhibitor therapy and risk of breast cancer recurrence in patients with rheumatoid arthritis: a nationwide cohort study. Ann Rheum Dis 74(12):2137-2143

10. Mercer LK, Low AS, Galloway JB, Watson KD, Lunt M, Symmons DP, Hyrich KL (2013) Anti-TNF therapy in women with rheumatoid arthritis with a history of carcinoma in situ of the cervix. Ann Rheum Dis 72(1):143-144

11. Elandt K, Aletaha D (2011) Treating rheumatic patients with a malignancy. Arthritis Res Ther 13:223

12. Bernatsky S, Clarke AE, Suissa S (2008) Hematologic malignant neoplasms after drug exposure in rheumatoidarthritis. Arch Intern Med 168:378-381

13. Wolfe F, Michaud K (2004) Lymphoma in rheumatoid arthritis: The effect of methotrexate and antitumor necrosis factor therapy in 18,572 patients. Arthritis Rheum 50:1740-1751

14. Buchbinder R, Barber M, Heuzenroeder L, Wluka AE et al (2008) Incidence of melanoma and other malignancies among rheumatoid arthritis patients treated with Methotrexate. Arthritis Care Res (Hoboken) 59(6):794-799

15. Scott Fl, Mamtani R, Brensinger C et al (2014) Risk of recurrent non-melanoma skin cancer with Methotrexate and anti-TNF use in rheumatoid arthritis. Arthritis Rheum 66(10 Suppl):1839

16. Solomon DH, Kremer JM, Fisher M et al (2014) Comparative cancer risk associated with methotrexate, other non-biologic and biologic diseasemodifying anti-rheumatic drugs. Semin Arthritis Rheum 43(4):489-497

17. Ruiz-Irastorza G, Ugarte A et al (2007) Antimalarials may influence the risk of malignancy in systemic lupus erythematosus. Ann Rheum Dis 66(6):815-817

18. Behrens F, Koehm M, Burkhardt H (2011) Update 2011: leflunomide in rheumatoid arthritis strengths and weaknesses. Curr Opin Rheumatol 23:282-287

19. Muellenhoff MW, Koo JY (2012) Cyclosporine and skin cancer: an international dermatologic perspective over 25 years of experience. A comprehensive review and pursuit to define safe use of cyclosporine in dermatology. J Dermatolog Treat 23(4):290-304

20. Deepak P, Sifuentes H, Sherid M et al (2013) T-cel non-Hodgkin's lymphomas reported to the FDA AERS with Tumor Necrosis Factor-alpha (TNF-a) inhibitors: results of the REFURBISH study. Am J Gastroenterol 108(1):99-105

21. Mariette $X$, Tubach F et al (2010) Lymphoma in patients treated with anti-TNF: results of the 3-year prospective French RATIO registry. Ann Rheum Dis 69(2):400-408

22. Mercer LK, Lunt M, Low ALS, Dixon WG et al (2015) Risk of solid cancer in patients exposed to anti-tumour necrosis factor therapy: results from the British Society for Rheumatology Biologics Register for Rheumatoid Arthritis. Ann Rheum Dis 74(6):1087-1093

23. Bongartz T, Sutton AJ, Sweeting MJ, Buchan I, Matteson EL, Montori V (2006) Anti-TNF antibody therapy in rheumatoid arthritis and the risk of serious infections and malignancies: systematic review and meta-analysis of rare harmful effects in randomized controlled trials. JAMA 295:2275-2285

24. Askling J, Fored CM, Brandt L, Baecklund E, Bertilsson L, Feltelius N, Coster L, Geborek P, Jacobsson LT, Lindblad S, Lysholm J, RantapaaDahlqvist S, Saxne T, Klareskog L (2005) Risks of solid cancers in patients with rheumatoid arthritis and after treatment with tumour necrosis factor antagonists. Ann Rheum Dis 64:1421-1426

25. Wolfe F, Michaud K (2007) Biologic treatment of rheumatoid arthritis and the risk of malignancy: analyses from a large US observational study. Arthritis Rheum 56:2886-2895

26. Dreyer L, Mellemkjær L et al (2013) Incidences of overall and site specific cancers in TNF a inhibitor treated patients with rheumatoid arthritis and other arthritides - a follow-up study from the DANBIO Registry. Ann Rheum Dis 72(1):79-82

27. Haynes K, Beukelman Tet al (2013) Tumor necrosis factor inhibitor therapy and cancer risk in chronic immune-mediated diseases. Arthritis Rheum 65(1):48-58

28. Long MD, Martin CF, Pipkin CA, Herfarth HH et al (2012) Risk of melanoma and nonmelanoma skin cancer among patients with inflammatory bowel disease. Gastroenterology 143(2):390-399

29. Mercer L, Askling J, Raaschou P et al (2014) No increased risk of developing a first invasive melanoma in rheumatoid arthritis patients treated with biologics: results of a collaborative project of 11 European biologics registers. Arthritis Rheum 66(10Suppl):Abstract 1838

30. Raaschou P, Simard JF, Asker-Hagelberg C et al (2014) Rheumatoid arthritis, anti-tumor necrosis factor therapy, and risk of squamous cell and basa cell skin cancer - a nationwide population based prospective cohort study from sweden. Arthritis Rheum 66(10):Abstract 846

31. Hooper M, Wenkert D, Bitman B, Dias VC, Bartley Y (2013) Malignancies in children and young adults on etanercept: summary of cases from clinical trials and post marketing reports. Pediatr Rheumatol Online J11(1):35

32. Klotsche J, Niewerth $M$, Haas JP, Huppertz HI, Zink A, Horneff G, Minden K (2015) Long-term safety of etanercept and adalimumab compared to methotrexate in patients with juvenile idiopathic arthritis (JIA). Ann Rheum Dis : doi:10.1136/ annrheumdis-annrheumdis-2014-206747

33. De la Forest M, Brugneaux J, Utard G, Salliot $C$ (2015) Safety of anti-TNFS in RA patients in real life: results from a systematic litterature review and meta-analyses from biologic registers. Ann Rheum Dis 74(Suppl2):702

34. van Vollenhoven RF, Emery Petal (2012) Long-term safety of rituximab in rheumatoid arthritis: 9.5-year follow-up of the global clinical trial programme with a focus on adverse events of interest in RA patients. Ann Rheum Dis 0:1-7

35. Matsuyama Y, Nagashima T et al (2011) Successful treatment of a patient with rheumatoid arthritis and lga-kappa multiple myeloma with Tocilizumab. Intern Med 50:639-642

36. Ogata A, Hirano T, Hishitani Y, Tanaka T (2012) Safety and efficacy of Tocilizumab for the treatment of rheumatoid arthritis. Arthritis Musculoskelet Disord 5:27-42

37. Genovese MC, Rubbert-Roth A et al (2013) Longterm safety and efficacy of Tocilizumab in patients with rheumatoid arthritis: a cumulative analysis of up to 4.6 years of exposure. JRheumato 40(6):768-780 
8. Weinblatt ME, Moreland LW et al (2013) Safety of abatacept administered intravenously in treatment of rheumatoid arthritis: integrated analyses of up to 8 years of treatment from the abatacept clinical trial program. J Rheumatol 40:6

39. Simon TA, Poncet C, Hochberg M et al (2015) Incidence rates of skin cancers during exposure to intravenous and subcutaneous abatacept in patients with rheumatoid arthritis: results from pooled clinical trial data. Ann Rheum Dis 74(Suppl 2):477

40. Salliot C, Beaudoin C, Chauffier K et al (2015) Prevalence and evolution of monoclonal gammopathy of undetermined significance in patients with rheumatoid arthritis treated with abatacept: data from the ORA registry. Ann Rheum Dis 74(Suppl2):445

41. Lopez-Olivo MA, Tayar JH et al (2012) Risk of Malignancies in Patients With Rheumatoid Arthritis Treated With Biologic Therapy. A Metaanalysis. JAMA 308(9):898-908

\section{Leitthemenübersicht}

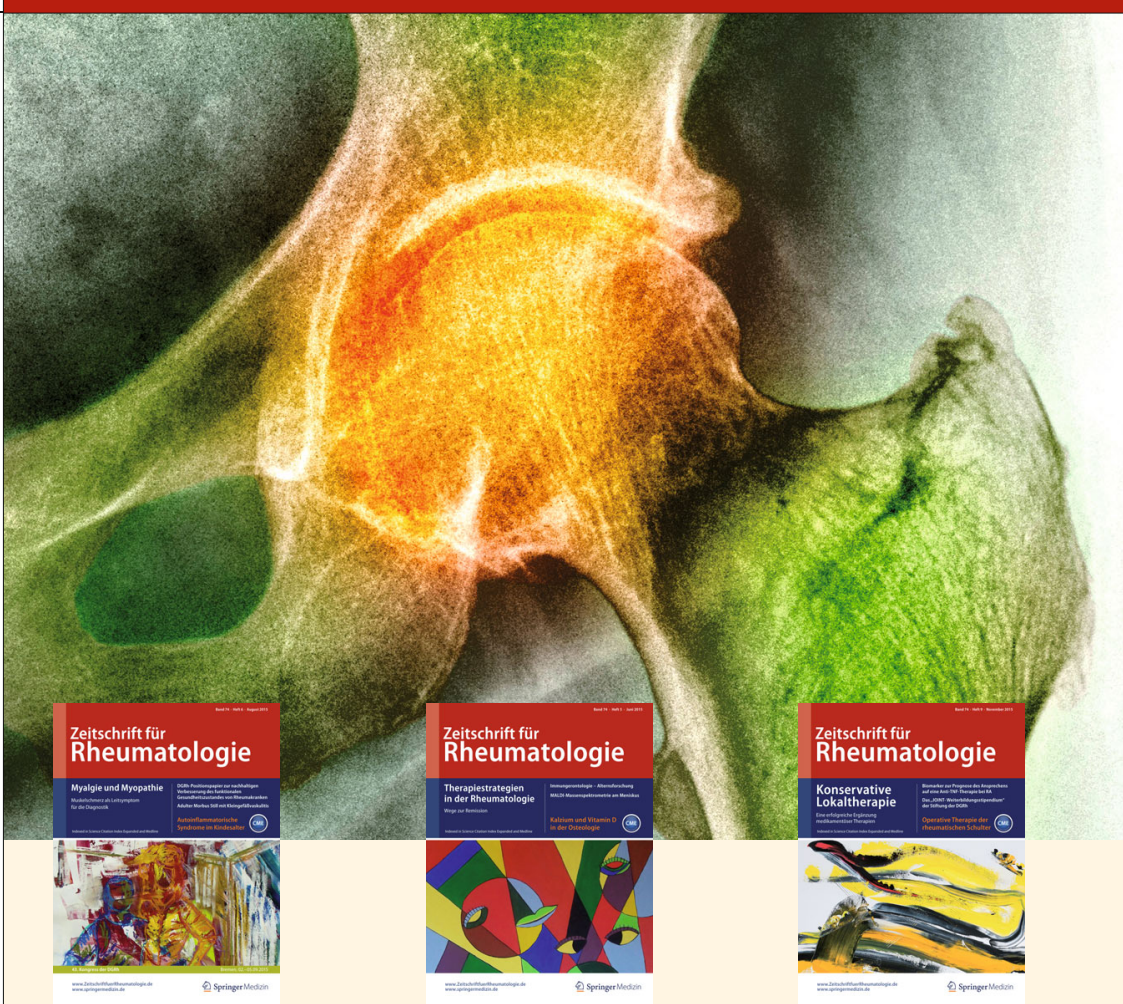

Die Zeitschrift für Rheumatologie bietet Ihnen jeden Monat umfassende und aktuelle Beiträge zu interessanten Themenschwerpunkten aus allen Bereichen der Rheumatologie.

\section{Überblick}

2015

01/15 Von der "Cellularpathologie" zur Zelltherapie

02/15 Neue Methoden für die klinische Forschung

03/15 Lupustherapie in Bewegung

04/15 Rheuma und Niere

05/15 Therapiestrategien in der Rheumatologie

06/15 Myalgie und Myopathien

07/15 Psychotherapeutische Therapieverfahren

08/15 Biosimilars

09/15 Konservative Lokaltherapie

10/15 Vaskulitiden

\section{Vorschau}

2016

01/16 Rheuma und Krebs

02/16 Seltene rheumatologische Krankheitsbilder

03/16 Fortschritte in der Kinderrheumatologie

04/16 Labordiagnostik

05/16 Osteoporose

06/16 Meilensteine in der Rheumatologie: 75 Jahre Innovation

07/16 lgG4-Syndrome

08/16 Erreger-induzierte Arthritiden

09/16 Transplantation bei rheumatischen Erkrankungen

10/16 „Rescue“-Therapieverfahren

Alle Inhalte der Zeitschrift für Rheumatologie finden Sie unter www.springermedizin.de/zeitschrift-fuer-rheumatologie

SpringerMedizin.de bietet Ihnen Zugang zu allen elektronisch verfügbaren Ausgaben Ihrer Zeitschrift - unabhängig davon, seit wann Sie die Zeitschrift abonniert haben.

Unser Tipp: Mit dem e.Med Kombi-Abo können Sie jederzeit auf alle Inhalte von SpringerMedizin.de zugreifen. AufWunsch erhalten sie darüber hinaus eine gedruckte Fachzeitschrift Ihrer Wahl.

Testen Sie e.Med 30 Tage lang kostenlos und unverbindlich!

Jetzt informieren unter

www.springermedizin.de/eMed

oder telefonisch unter 0800-77 80777 (Montag bis Freitag, 10 bis $17 \mathrm{Uhr}$ ) 